\title{
OPE and quark-hadron duality for two-point functions of tetraquark currents in the $1 / N_{c}$ expansion
}

\author{
Wolfgang Lucha $\odot,{ }^{1}$ Dmitri Melikhov $\odot,{ }^{2,3,4}$ and Hagop Sazdjian $\oplus^{5}$ \\ ${ }^{1}$ Institute for High Energy Physics, Austrian Academy of Sciences, \\ Nikolsdorfergasse 18, A-1050 Vienna, Austria \\ ${ }^{2}$ D. V. Skobeltsyn Institute of Nuclear Physics, M. V. Lomonosov Moscow State University, \\ 119991 Moscow, Russia \\ ${ }^{3}$ Joint Institute for Nuclear Research, 141980 Dubna, Russia \\ ${ }^{4}$ Faculty of Physics, University of Vienna, Boltzmanngasse 5, A-1090 Vienna, Austria \\ ${ }^{5}$ Université Paris-Saclay, CNRS/IN2P3, IJCLab, 91405 Orsay, France
}

(Received 7 December 2020; accepted 24 December 2020; published 12 January 2021)

\begin{abstract}
We discuss the operator product expansion (OPE) and quark-hadron duality for two-point Green functions of tetraquark currents. We emphasize that the factorizable part of the OPE series for such Green functions, including nonperturbative contributions described by QCD condensates, is saturated by the full system of ordinary hadrons and therefore cannot have any relationship to the possible tetraquark bound states. Possible tetraquark bound states may be contained in nonfactorizable parts of these Green functions. In the framework of the $1 / N_{c}$ expansion in $\operatorname{QCD}\left(N_{c}\right)$, nonfactorizable parts of the two-point Green functions of tetraquark currents provide $N_{c}$-suppressed contributions compared to the $N_{c}$-leading factorizable parts. A possible exotic tetraquark state may appear only in $N_{c}$-subleading contributions to the QCD Green functions, in full accord with the well-known rigorous properties of large- $N_{c}$ QCD.
\end{abstract}

DOI: $10.1103 /$ PhysRevD.103.014012

\section{INTRODUCTION}

The correlation functions of two local colorless currents are the simplest gauge-invariant Green functions that have a unique decomposition in terms of the physical hadron states. These correlation functions are defined as vacuum expectation values of the time-ordered products of two local gauge-invariant quark currents taken at different locations:

$$
\Pi\left(p^{2}\right)=i \int d^{4} x e^{i p x}\left\langle 0\left|T\left\{j(x) j^{\dagger}(0)\right\}\right| 0\right\rangle .
$$

We shall discuss and compare two cases: $j$ being a local bilinear quark current and $j$ being a tetraquark current. The Dirac structure of the currents will be of no relevance for our arguments and will not be specified; we therefore do not explicitly write the appropriate combinations of Dirac matrices between the quark fields. The Wilson operator product expansion (OPE) [1] provides the following expansion for the $T$-product:

Published by the American Physical Society under the terms of the Creative Commons Attribution 4.0 International license. Further distribution of this work must maintain attribution to the author(s) and the published article's title, journal citation, and DOI. Funded by SCOAP ${ }^{3}$.
$T\left\{j(x) j^{\dagger}(0)\right\}=C_{0}\left(x^{2}, \mu\right) \hat{1}+\sum_{n} C_{n}\left(x^{2}, \mu\right): \hat{O}_{n}(x=0, \mu):$

where $\mu$ is a renormalization scale. The two-point function (for those cases where only light quarks are involved) is then expanded in the form

$\Pi\left(p^{2}\right)=\Pi_{\text {pert }}\left(p^{2}, \mu\right)+\sum_{n} \frac{C_{n}}{\left(p^{2}\right)^{n}}\left\langle 0\left|: \hat{O}_{n}(x=0, \mu):\right| 0\right\rangle$.

The QCD vacuum is nonperturbative, and its properties are characterized by the condensates - nonzero vacuum expectation values of gauge-invariant operators, depending, in general, on the renormalization scale $\mu,\langle 0|: \hat{O}(0, \mu):| 0\rangle \neq 0$ [2-5]. Hereafter, we denote $\langle 0|\ldots| 0\rangle \equiv\langle\ldots\rangle$. In the OPE context, perturbative diagrams describe the contribution of the unit operator. The Wilson coefficients of the local operators : $\hat{O}(0, \mu)$ : are obtained from perturbative diagrams according to known rules [3]. The appropriate diagrams will be displayed below; we show only diagrams containing quark and gluon lines but do not show those diagrams that contain Faddeev-Popov ghosts.

It proved efficient to generalize QCD, based on the color gauge group $\mathrm{SU}(3)$, to the case of the color gauge group 
$\mathrm{SU}\left(N_{c}\right)$ and to consider the $1 / N_{c}$ expansion of the Green functions in $\mathrm{QCD}\left(N_{c}\right)$ in the so-called 't Hooft limit, the strong coupling constant scaling as $\alpha_{s} \sim 1 / N_{c}$ [6]. For the discussion of exotic states of any structure [in $\operatorname{QCD}\left(N_{c}\right)$ one may have a very rich structure of colorless hadron states] the following features of large- $N_{c}$ QCD are of special significance: as shown by Witten [7], large- $N_{c}$ Green functions are saturated by noninteracting ordinary mesons. This means, in particular, that any possible exotic states may appear only in $N_{c}$-subleading contributions to the $\operatorname{QCD}\left(N_{c}\right)$ Green functions [7,8]. This is a distinguishing property of exotic tetraquark mesons compared to ordinary quark-antiquark mesons: the latter appear already in the $N_{c}$-leading parts of the $\mathrm{QCD}\left(N_{c}\right)$ Green functions.

The possibility of the existence of narrow exotic states in QCD at large $N_{c}$ has been studied in recent years in a number of publications [9-20]. The present paper makes use of large- $N_{c}$ arguments in a slightly different context: we study two-point functions of bilinear quark currents and of tetraquark currents and discuss the appearance of the tetraquark bound states in the latter, giving further theoretical arguments in the derivation of the tetraquarkadequate ( $T$-adequate) sum rules, formulated in our recent publications $[21,22]$. Here, we make the following new steps:

(i) We show that the $T$-adequate sum rules in $\mathrm{SU}\left(N_{c}\right)$ should be based on appropriate nonfactorizable parts of the OPE for two-point functions of the tetraquark currents. In this way, the tetraquark contributions are compatible with the well-known rigorous property of QCD at large $N_{c}$ : the $N_{c}$-leading Green functions are saturated by the ordinary mesons; any exotic states may appear only in $N_{c}$-subleading contributions.

(ii) We discuss nonperturbative effects in two-point functions of tetraquark currents [our analyses $[21,22]$ considered perturbative diagrams and did not address nonperturbative effects] and identify those condensate contributions that appear in the $T$-adequate duality relations and $T$-adequate sum rules.

The paper is organized as follows: section II recalls the large- $N_{c}$ behavior of the two-point Green functions of bilinear quark currents and compares the OPE with the hadron saturation of these Green functions. Section III studies the OPE for the two-point function of tetraquark currents including nonperturbative condensate contributions and discusses the quark-hadron duality relations that may involve possible tetraquark states. Section IV presents our conclusions and outlook.

\section{TWO-POINT FUNCTION OF BILINEAR QUARK CURRENTS}

Let us start with some useful algebraic relations for the group $\mathrm{SU}\left(N_{c}\right)$ [23]. The generators $T^{A}, A=1, \ldots, N_{c}^{2}-1$, considered in the fundamental representation, satisfy the color Fierz rearrangement

$$
\left(T^{A}\right)_{i i^{\prime}}\left(T^{A}\right)_{j j^{\prime}}=\frac{1}{2} \delta_{i j^{\prime}} \delta_{i^{\prime} j}-\frac{1}{2 N_{c}} \delta_{i i^{\prime}} \delta_{j j^{\prime}},
$$

where the generators are normalized according to

$$
\operatorname{Tr}\left(T^{A} T^{B}\right)=\frac{1}{2} \delta^{A B}
$$

The relation (2.1) suggests that, with respect to counting an overall $N_{c}$-leading color factor of a Feynman diagram, any gluon line may be replaced by a $\bar{q} q$ double line. To calculate the $N_{c}$-subleading terms in the expansion of a Green function, one has to take into account, in the gluon lines, the second term of the right-hand side of Eq. (2.1).

We now briefly recall the properties of the $1 / N_{c^{-}}$ expansion of the OPE series for bilinear quark currents, $j=\bar{q} q$.

\section{A. Perturbative diagrams}

Figure 1 shows diagrams according to their behavior in the framework of the $1 / N_{c}$ expansion, assuming that the strong coupling constant scales as $\alpha_{s} \sim 1 / N_{c}$. When calculating the $1 / N_{c}$ behavior of a diagram, we replace the gluon line by a double $\bar{q} q$ line. Doing so, we pick up the leading behavior at large $N_{c}$, but omit corrections of the order $1 / N_{c}^{2}$. [The second term in Eq. (2.1) contains a factor $1 / N_{c}$ and, in addition, the number of color loops generated by the second term is reduced by one compared to the first term, thus yielding an overall suppression factor $\left.1 / N_{c}^{2}\right]$. So, all diagrams with a specific large- $N_{c}$ behavior also generate contributions to lower orders of the $1 / N_{c}$ expansion. For instance, the $O\left(N_{c}\right)$ diagrams in Fig. 1(a) also generate contributions to diagrams of the order $O\left(N_{c}^{-1}\right)$. The $N_{c^{-}}$ leading perturbative diagrams of Fig. 1(a) are planar diagrams without sea-quark loops. Using the language of intermediate states, these diagrams can be identified as those diagrams that have intermediate valence $\bar{q} q$ states plus an arbitrary number of gluons; the $N_{c}$-leading perturbative QCD diagrams do not have cuts corresponding to four quarks and an arbitrary number of gluons, six quarks and an arbitrary number of gluons, etc. Diagrams with multiquark intermediate states have an $N_{c}$-subleading behavior.

\section{B. Power corrections}

Power corrections are shown in Fig. 2: the Wilson coefficients describing the contribution of the appropriate operators may be obtained from the perturbative diagrams of Fig. 1 by breaking one or more quark and gluon lines. For instance, the diagram of Fig. 2(a) provides the Wilson coefficient of the operator $\bar{q} q$; the diagram of Fig. 2(b) 


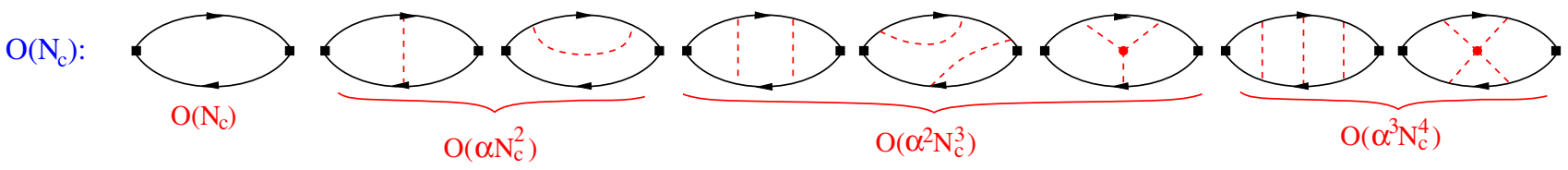

(a)
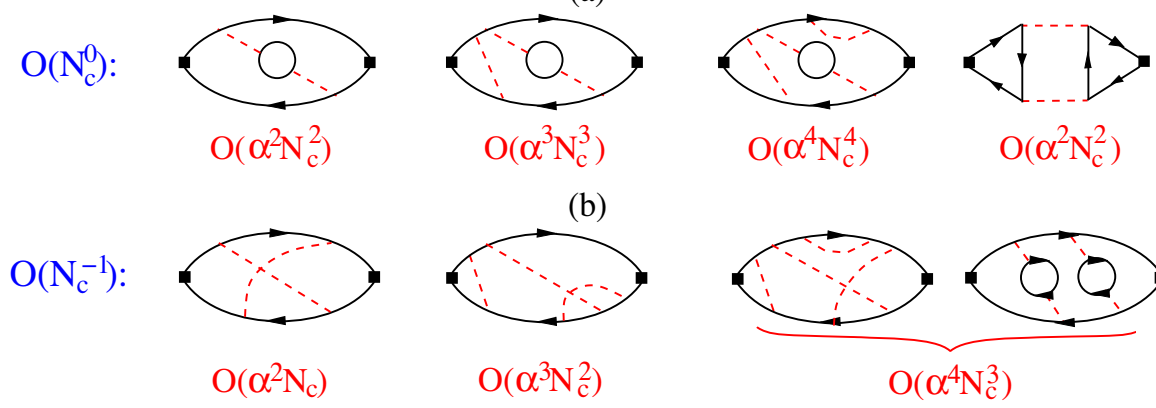

(b)

(c)

FIG. 1. Perturbative series for the two-point function of bilinear quark currents and its classification in powers of $1 / N_{c}$. (a) $O\left(N_{c}\right) . N_{c^{-}}$ leading diagrams are diagrams of planar topology without sea-quark loops (i.e., containing only the loop of valence quarks that enter the interpolating current) and with an arbitrary number of planar gluon exchanges. No annihilation-type diagrams appear at this $N_{c}$-order. Taking into account that $\alpha_{s} \sim 1 / N_{c}$, they have the overall dependence $O\left(N_{c}\right)$. (b) $O\left(N_{c}^{0}\right)$. Diagrams with the $N_{c}$-leading contribution of this order are shown; these diagrams are (i) planar diagrams with one sea-quark loop and an arbitrary number of gluon exchanges or (ii) quark-annihilation diagrams. (c) $O\left(N_{c}^{-1}\right)$. Diagrams with $N_{c}$-leading behavior of this order are of two different classes: (i) nonplanar diagrams with one gluonic handle and an arbitrary number of planar gluon exchanges but no sea-quark loops and (ii) planar diagrams containing two sea-quark loops and an arbitrary number of planar gluon exchanges.

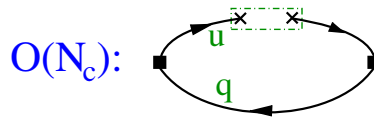

(a)

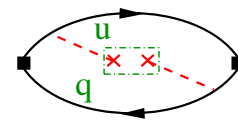

(b)

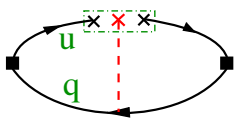

(c)

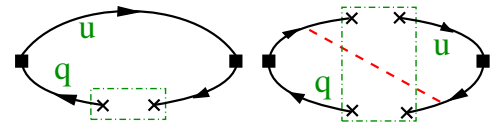

(d)

FIG. 2. Nonperturbative power corrections in $\left\langle j j^{\dagger}\right\rangle$ of bilinear quark currents: (a) contribution of dimension-3 quark condensate $\langle\bar{u} u\rangle$; (b) contribution of dimension-4 gluon condensate $\left\langle\alpha_{s} G G\right\rangle$; (c) contribution of dimension-5 mixed condensate $\left\langle\bar{u} \sigma_{\mu \nu} G^{\mu \nu} u\right\rangle$, with $\sigma_{\mu \nu}=\frac{1}{2 i}\left[\gamma_{\mu}, \gamma_{\nu}\right]$, the $\gamma$ s being the Dirac matrices; (d) contributions emerging if $q$ is the light quark: that of dimension-3 quark condensate $\langle\bar{q} q\rangle$ and that of dimension-6 four-quark condensate $\langle\bar{u} u \bar{q} q\rangle$.

gives the Wilson coefficient of the operator $G G$, where $G$ is the gluon field strength, etc.

Power corrections scale with $N_{c}$ as follows:

$$
\langle\bar{q} q\rangle \sim N_{c} ; \quad\left\langle\alpha_{s} G G\right\rangle \sim N_{c} ; \quad\langle\bar{q} q \bar{q} q\rangle \sim N_{c}^{2} .
$$

Obviously, nonperturbative effects described by the condensates contribute on the same footing as perturbative effects at each order in $1 / N_{c}$. So, even at $N_{c}$-leading order QCD is not fully perturbative: although the strong coupling may be made arbitrarily small, nonperturbative effects described by the condensates do not disappear and survive the large- $N_{c}$ limit.

\section{Hadron saturation of two-point function and sum rules}

As is well known $[7,8]$, the spectrum of states of $\mathrm{QCD}\left(N_{c}\right)$ in the limit $N_{c} \rightarrow \infty$ contains towers of an infinite number of free, stable and noninteracting mesons. Meson-meson elastic scattering amplitudes are of order $1 / N_{c}$, and the decay amplitudes of mesons into two mesons are of order $1 / N_{c}^{1 / 2}$. The notion of valence quarks takes in the above limit a precise meaning. Mesons are made of pure $\bar{q} q$ states, rather than of $\bar{q} \bar{q} q q$ states, which only appear at subleading orders of $N_{c}$. Conversely, states whose $N_{c^{-}}$ leading element is composed of $\bar{q} \bar{q} q q$ states correspond to two-meson states. These properties allow us to make a systematic correspondence between the OPE and the hadron saturation of two-point correlation functions.

Let us consider the two-point function of the elastic vector current, $V_{\mu}=\bar{q} \gamma_{\mu} q$, and denote it as $\Pi_{\mu \nu}^{V}(x) \equiv$ $\left\langle T\left\{V_{\mu}(x) V_{\nu}(0)\right\}\right\rangle$. Obviously, we have light pseudoscalar mesons (hereafter referred to as pions). Figures 3(a) and 3(b) show the scaling of the hadron diagrams and vertices at large $N_{c}$, and Fig. 3(c) shows the $N_{c}$-leading sum rule: the sum over stable vector mesons is dual to the 

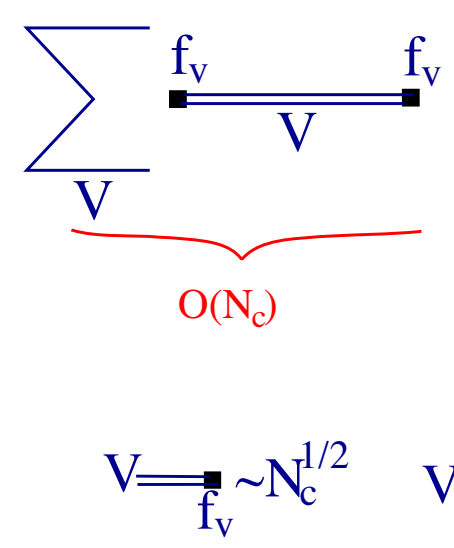
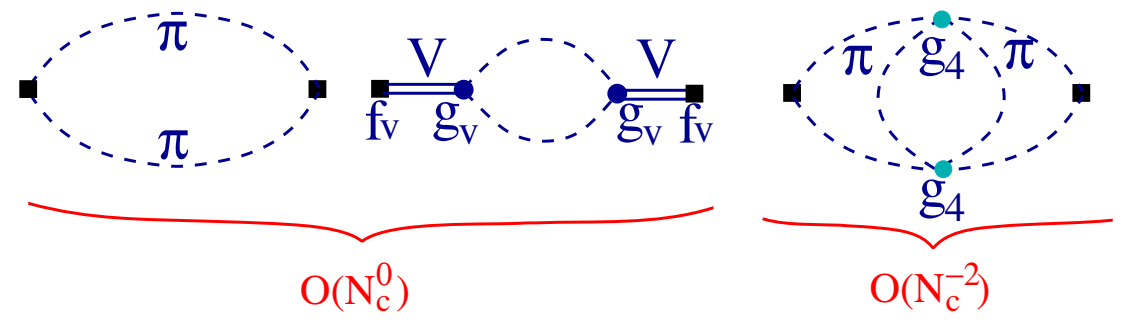

(a)
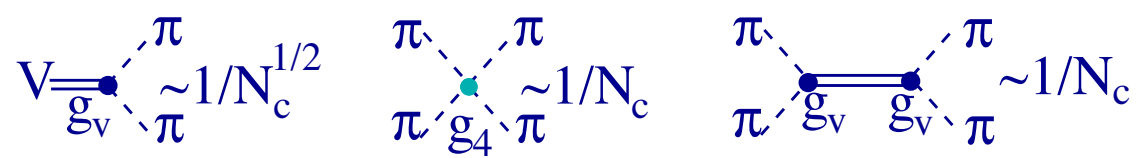

(b)

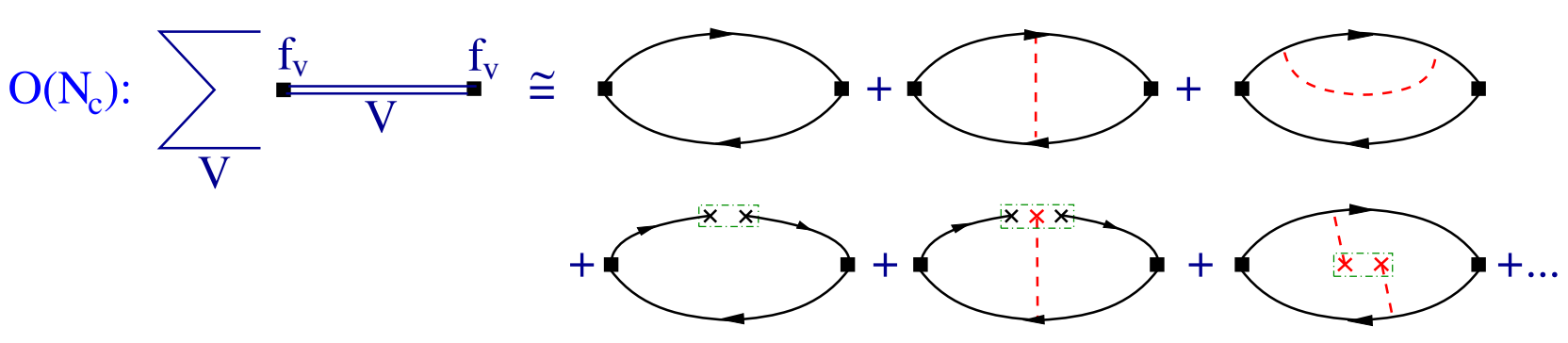

(c)

FIG. 3. Duality relation for the elastic vector two-point function at large $N_{c}$. (a) Typical hadron diagrams emerging in the hadron representation of $\Pi_{V}$. (b) Scaling of the hadron couplings at large $N_{c}$. (c) $N_{c}$-leading sum rule [of order $O\left(N_{c}\right)$ ].

$N_{c^{-}}$leading OPE. Obviously, one can include some $N_{c^{-}}$ subleading effects on the hadron and/or on the OPE side of this sum rule. However, for the consistency of the full approach, it is mandatory that the $N_{c}$-leading contribution on the hadron side matches the $N_{c}$-leading contribution on the OPE side. In fact, the vector sum rule in real QCD [2] looks very similar to the relation shown in Fig. 3(c) and thus perfectly satisfies the large- $N_{c}$ consistency.

Closing this discussion, we notice that the correlation function of bilinear quark currents describes the "minimal" colorless cluster and thus does not contain any other factorizable colorless clusters. As a result, the hadron saturation of the $N_{c}$-leading part of the correlation function contains all intermediate hadron states with the appropriate quantum numbers, starting from the one-meson state.

\section{OPE FOR CORRELATORS OF TETRAQUARK CURRENTS}

Let us now turn to two-point functions of tetraquark currents. We consider four-quark currents consisting of two antiquarks of generic flavors $b$ and $c$ and two quarks of generic flavors $u$ and $d$. For the sake of argument, we make two assumptions: first, we assume that all quark flavors are different-this simplifies the topology of the appropriate QCD diagrams, avoiding, in particular, the discussion of quark annihilation diagrams. Our second assumption is that the two antiquarks, $\bar{b}$ and $\bar{c}$, are heavy and therefore do not produce quark condensates; the two quarks, $u$ and $d$, are light and therefore develop local vacuum condensates (quark condensates $\langle\bar{u} u\rangle,\langle\bar{d} d\rangle$, mixed quark-gluon condensates $\left\langle\bar{u} \sigma_{\mu \nu} T^{A} G_{\mu \nu}^{A} u\right\rangle,\left\langle\bar{d} \sigma_{\mu \nu} T^{A} G_{\mu \nu}^{A} d\right\rangle$, four-quark condensates $\langle\bar{u} u \bar{d} d\rangle$, etc.). These assumptions simplify the discussion but do not change any essential qualitative feature of our analysis.

As follows from the property of cluster reducibility of multiquark operators [24], any gauge-invariant multiquark operator can be reduced to a combination of products of colorless clusters. In our case of the $\bar{b} \bar{c} u d$ flavor content of the tetraquark current, colorless clusters of two different flavor structures emerge in QCD (see $[25,26]$ ):

$$
\theta_{\bar{b} u \bar{c} d}=j_{\bar{b} u} j_{\bar{c} d}, \quad \theta_{\bar{b} d \bar{c} u}=j_{\bar{b} d} j_{\bar{c} u},
$$

with $j_{\bar{a} f}=\bar{q}_{a} q_{f}$. We therefore should distinguish between the diagrams where quark flavors in the initial and final states are combined in the same way (direct diagrams) and in a different way (quark-exchange or recombination diagrams). The Feynman diagrams for the corresponding four-point functions have different topologies and structures of their four-quark singularities and therefore require 


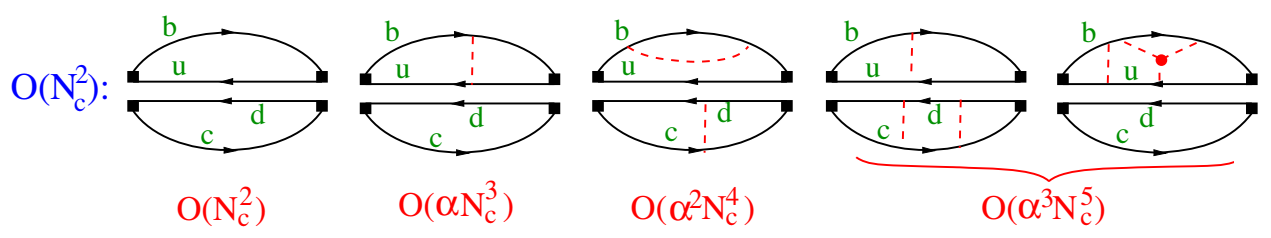

(a)

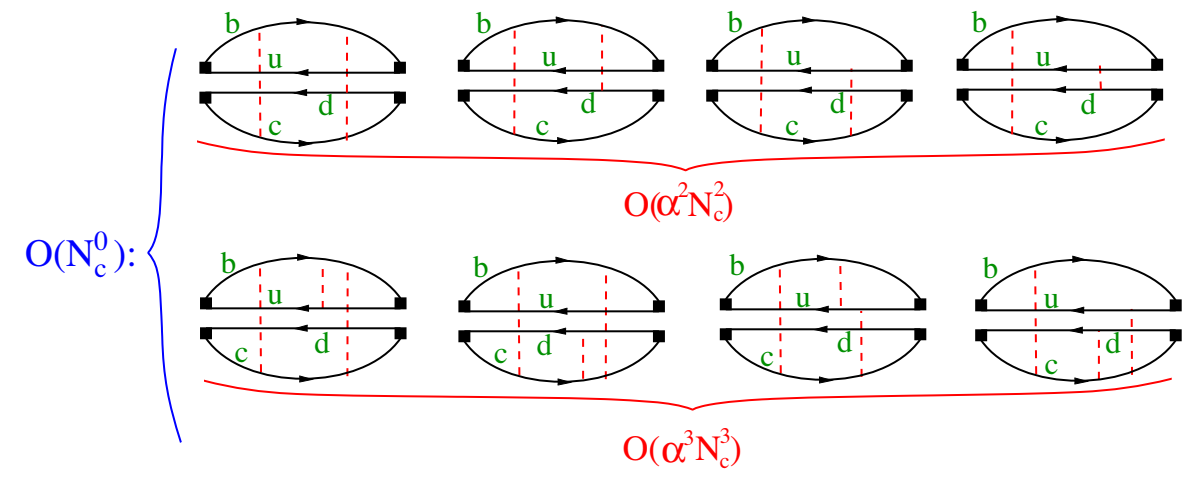

(b)

FIG. 4. Typical perturbative diagrams emerging in the OPE for $\Pi^{\text {dir }}$. (a) Planar diagrams with an arbitrary number of planar gluon exchanges inside the $b u$ or $c d$ quark loops, whereas there are no gluon exchanges between the quark loops. These diagrams have a factorizable structure, i.e., may be represented, in coordinate space, as a product of two expressions-one corresponding to the $b u$ loop and the other corresponding to the $c d$ loop. They have the behavior $\sim N_{c}^{2}$. (b) Diagrams with two-gluon exchanges between the loops $b u$ and $c d$; the two gluons may be attached to quarks/antiquarks in different quark loops in any combination. Such diagrams have a cylinder topology and behave as $N_{c}^{0}$ at large $N_{c}$. Adding an arbitrary number of planar gluon exchanges inside the loops $b u$ or $c d$ does not change the large- $N_{c}$ scaling behavior.

separate analyses [21,22]. Here, we discuss the direct Green function

$$
\Pi^{\operatorname{dir}}(x) \equiv\left\langle T\left\{\theta_{\bar{b} u \bar{c} d}(x) \theta_{\bar{b} u \bar{c} d}^{\dagger}(0)\right\}\right\rangle
$$

\section{A. Perturbative diagrams}

Figure 4 shows perturbative diagrams in the OPE for $\Pi^{\mathrm{dir}}$ with different types of gluon exchanges.

Perturbative diagrams without gluon exchanges between the quark loops have a factorizable structure, i.e., factorize, in coordinate space, into the product of two-point functions of bilinear quark currents, $\Pi_{\bar{b} u}(x) \Pi_{\bar{c} d}(x)$, where

$$
\begin{aligned}
\Pi_{\bar{b} u}(x) & \equiv\left\langle T\left\{j_{\bar{b} u}(x) j_{\bar{b} u}^{\dagger}(0)\right\}\right\rangle, \\
\Pi_{\bar{c} d}(x) & \equiv\left\langle T\left\{j_{\bar{c} d}(x) j_{\bar{c} d}^{\dagger}(0)\right\}\right\rangle .
\end{aligned}
$$

One can refer to these diagrams as "disconnected" diagrams, bearing in mind, however, that the vertices corresponding to the initial (final) bilinear currents are connected to each other. ${ }^{1}$ So the term "disconnected" should apply to the internal parts of the diagrams. We will prefer to term these diagrams "factorizable." Since any of the quark loops behave as $\sim N_{c}$, the factorizable diagrams are of order $N_{c}^{2}$.

\footnotetext{
${ }^{1}$ Notice that the terms "connected" and "disconnected" in lattice calculations have quite different meanings; see, e.g., [11].
}

Factorizable diagrams can be isolated in a unique way and provide the $N_{c}$-leading behavior of $\Pi^{\mathrm{dir}}(x)$.

In the diagrams of Fig. 4(b) two quark loops talk to each other via gluon exchanges. Since both quark loops represent colorless clusters, one needs at least two gluons to be exchanged between the loops. Diagrams with two gluon exchanges between the loops and an arbitrary number of planar gluon exchanges inside each of the loops $b u$ and $c d$ have cylinder topology; see Figs. 1 and 2 of Ref. [20]. Their behavior at large $N_{c}$ is $N_{c}^{0}$.

One can also have three or more gluon exchanges between the quark loops. All these diagrams have a topology of a cylinder with a number of handles. Each handle reduces the large- $N_{c}$ behavior by two powers of $N_{c}$.

\section{B. Diagrams containing condensates}

Diagrams containing condensates may be obtained from the perturbative diagrams by breaking the internal quark and gluon lines and sending the corresponding particles to vacuum condensates.

Let us start with the factorizable perturbative diagrams of Fig. 4(a). By breaking a light-quark or a gluon line in these diagrams, one obtains the contributions of condensates of lowest dimensions, the quark condensate $\langle\bar{q} q\rangle$, the gluon condensate $\left\langle\alpha_{s} G G\right\rangle$, or the mixed quark-gluon condensate, shown in Fig. 5(a). These diagrams have the same large- $N_{c}$ behavior, $O\left(N_{c}^{2}\right)$, as the original perturbative diagrams of 


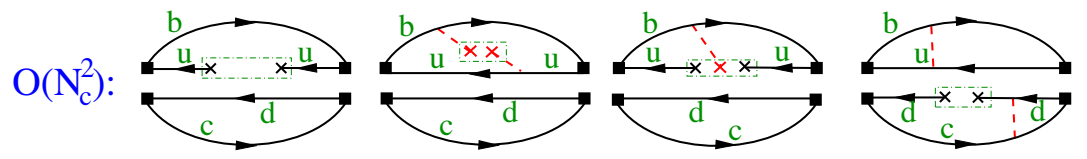

(a)

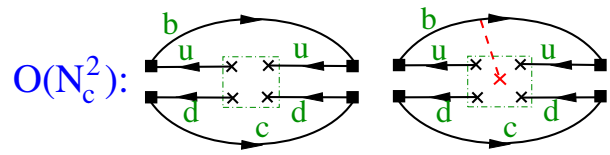

(b)

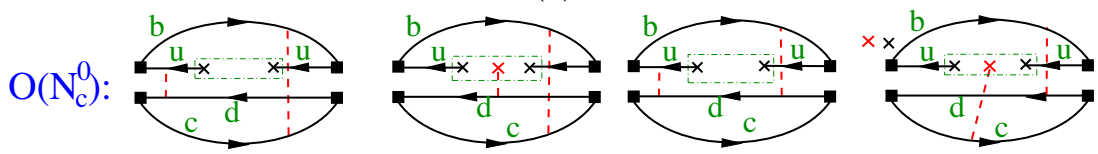

(c)

FIG. 5. Typical diagrams belonging to different classes containing condensate contributions in the OPE for $\Pi^{\text {dir }}$. (a) Factorizable diagrams obtained by inserting condensate contributions in perturbative diagrams of Fig. 4(a). (b) Power corrections of the mixed type: they are proportional to condensates of dimension- 6 or higher $(\langle\bar{u} u \bar{d} d\rangle$, etc.) and obtained by sending quark or gluon fields from different quark loops of factorizable perturbative diagrams of Fig. 4(a) to vacuum condensates. In the four-quark (and higher) condensates, factorizable and nonfactorizable parts may be isolated; see Fig. 6. (c) Power corrections of nonfactorizable type: they are obtained by sending to the condensate quarks and gluons in nonfactorizable diagrams of Fig. 4(b).

Fig. 4(a). Important for us is that diagrams containing the quark, the gluon, and the mixed quark-gluon condensates are of the factorizable type, which is the same as that of the original perturbative diagrams.

A new feature emerges when one calculates the contributions (i.e., the Wilson coefficients) of higherdimension four-quark and four-quark-gluon condensates; see Fig. 5(b).

Here, both light quarks, from the upper and the lower loops, can be sent to the condensate simultaneously. For further analysis, it is convenient to isolate factorizable contributions from higher-dimension condensates. For instance, the four-quark condensate may be split into factorizable and nonfactorizable (NF) parts in a unique way, see Fig. 6:

$$
\langle\bar{u} u \bar{d} d\rangle \equiv\langle\bar{u} u\rangle\langle\bar{d} d\rangle+\langle\bar{u} u \bar{d} d\rangle_{\mathrm{NF}} .
$$

The relevance of isolating factorizable parts out of the condensates of higher dimensions will become clear shortly.

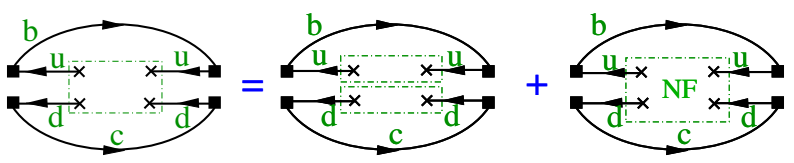

FIG. 6. Splitting of the condensates of higher dimensions into factorizable and NF parts. The factorizable parts provide the $N_{c^{-}}$ leading contribution, whereas the nonfactorizable pieces have an $N_{c}$-subleading behavior. For instance, $\langle\bar{u} u\rangle \sim N_{c},\langle\bar{d} d\rangle \sim N_{c}$, $\langle\bar{u} u \bar{d} d\rangle \sim N_{c}^{2}$, whereas $\langle\bar{u} u \bar{d} d\rangle_{\mathrm{NF}} \sim N_{c}^{0}$.

\section{Isolating the factorizable part from the OPE for two-point functions of tetraquark currents $\Pi^{\text {dir }}$}

We are now fully prepared to isolate the factorizable part from the OPE for the direct two-point function of the tetraquark currents $\Pi^{\text {dir }}$, including both perturbative and nonperturbative condensate contributions. At the level of diagrams, one may naively suspect the following decomposition of the OPE for $\Pi^{\text {dir }}$ shown in Fig. 7:

$$
\Pi^{\mathrm{dir}}(x)=\Pi_{\bar{b} u}(x) \Pi_{\bar{c} d}(x)+\Pi_{\mathrm{NF}, 1}^{\mathrm{dir}}(x)+\Pi_{\mathrm{NF}, 2}^{\mathrm{dir}}(x) .
$$

As we shall see, this formula contains double counting of some of the nonperturbative contributions, and the correct decomposition is a bit more tricky.

The factorizable part, $\Pi_{\bar{b} u}(x) \Pi_{\bar{c} d}(x)$, including the appropriate nonperturbative condensate contributions, is obvious and is shown in Fig. 7(a).

More cumbersome are NF contributions. Here, we encounter two types of such NF contributions:

(i) $\Pi_{\mathrm{NF}, 1}^{\mathrm{dir}}(x)$, shown in Fig. 7(b), describes nonfactorizable parts of the condensates of higher dimension corresponding to Fig. 5(b); somewhat tricky, these NF nonperturbative power corrections are generated by factorizable perturbative diagrams of Fig. 4(a). Radiative corrections due to gluon exchanges inside the loops $b u$ and $c d$ (and not between the loops) are included in this class of NF contributions.

To understand the proper way to take into account such contributions, let us recall that nonzero vacuum condensates emerge due to interactions with the nonperturbative soft gluon fields. If we look into the anatomy of $\langle\bar{u} u \bar{d} d\rangle_{\mathrm{NF}}$, this quantity is nonzero due 


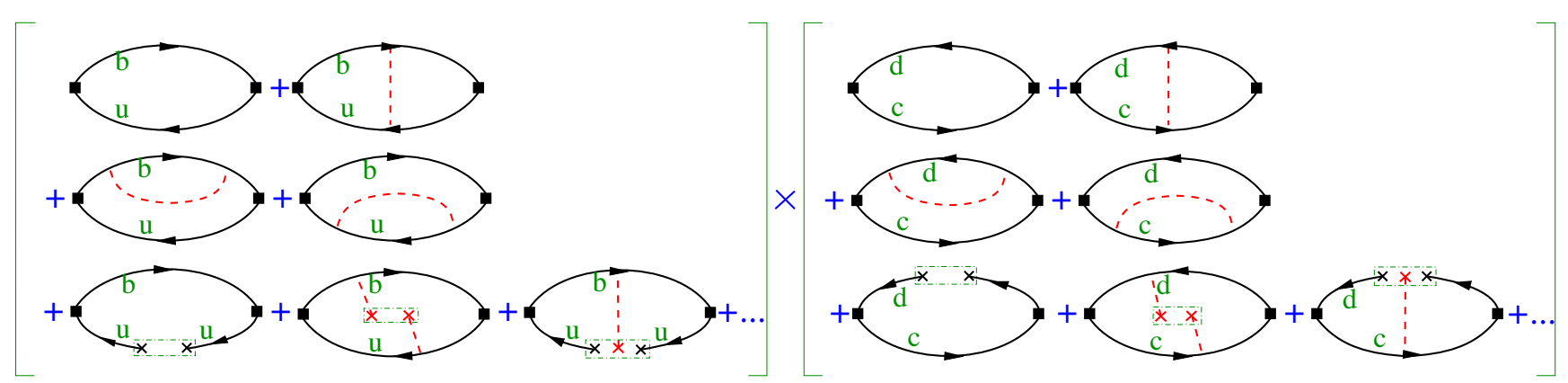

(a)

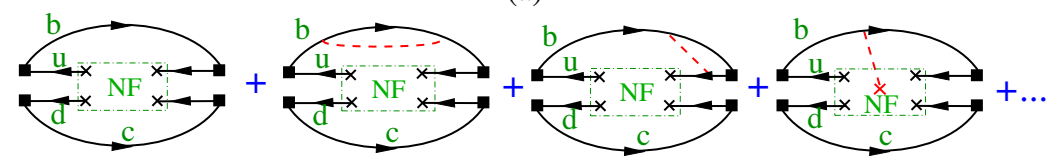

(b)

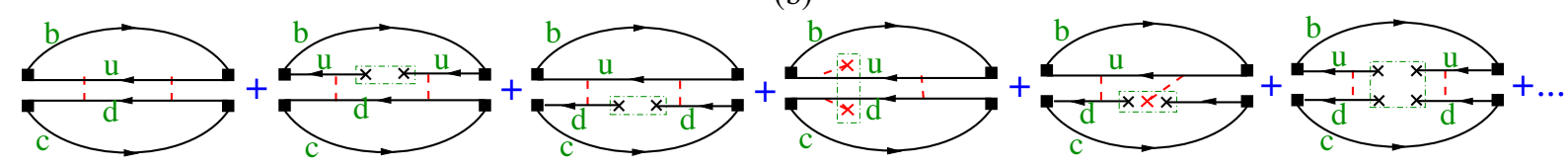

(c)

FIG. 7. Different types of contributions to the OPE for $\Pi^{\text {dir }}$ corresponding to the decomposition (3.5). (a) Factorizable part of the OPE given by the product of $\Pi_{\bar{b} u}(x)$ and $\Pi_{\bar{c} d}(x) ; \Pi_{\bar{b} u}(x)$ and $\Pi_{\bar{c} d}(x)$ here contain both perturbative and nonperturbative condensate contributions. (b) Typical diagrams for the nonfactorizable contribution $\Pi_{\mathrm{NF}, 1}^{\mathrm{dir}}(x)$. (c) Nonfactorizable contribution $\Pi_{\mathrm{NF}, 2}^{\mathrm{dir}}(x)$. Only those diagrams where both gluons are exchanged between the quark $u$ of the $b u$ loop and the quark $d$ of the $d c$ loop are displayed. Diagrams corresponding to other gluon exchanges between the $b u$ and $c d$ quark loops and the appropriate power corrections can be easily drawn.

to the interactions with the soft gluons of the type shown in Fig. 8. Precisely the same nonperturbative corrections emerge in the nonperturbative contributions related to the diagrams of Fig. 7(c) where gluons are exchanged between the $u$ quark of the $b u$ quark loop and the $d$ quark of the $c d$ quark loop. (This actually explains the fact that the large- $N_{c}$ behaviors of $\langle\bar{q} q\rangle^{2}$ and $\langle\bar{q} q \bar{q} q\rangle_{\mathrm{NF}}$ differ from each other.)

Taking into account both effects described by $\Pi_{\mathrm{NF}, 1}^{\mathrm{dir}}(x)$ and by nonperturbative corrections in those parts of $\Pi_{\mathrm{NF}, 2}^{\mathrm{dir}}(x)$ which correspond to two-gluon exchanges between $u$ and $d$ quarks from the different quark loops would be a double counting. We therefore take the appropriate nonperturbative contributions into account as a part of $\Pi_{\mathrm{NF}, 2}^{\mathrm{dir}}(x)$ and omit $\Pi_{\mathrm{NF}, 1}^{\mathrm{dir}}(x)$.

(ii) $\Pi_{\mathrm{NF}, 2}^{\mathrm{dir}}(x)$, Fig. 7(c), describes the "genuinely" nonfactorizable perturbative diagrams of Fig. 4(b) and

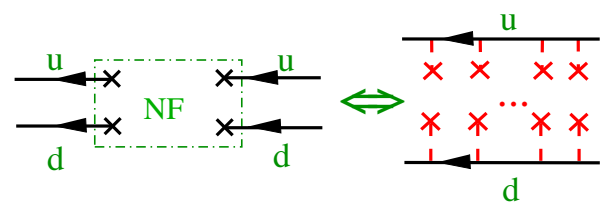

FIG. 8. Emergence of the nonfactorizable part of the four-quark condensate $\langle\bar{u} u \bar{d} d\rangle$ through interactions with the nonperturbative gluon background. power corrections of Fig. 5(c); the latter are obtained via the conventional rules by breaking the propagating lines of light quarks and gluons in the perturbative diagrams of Fig. 4(b).

In the end, the proper decomposition of $\Pi^{\text {dir }}$ that avoids the double counting of the nonperturbative corrections has the form

$$
\Pi^{\operatorname{dir}}(x)=\Pi_{\bar{b} u}(x) \Pi_{\bar{c} d}(x)+\Pi_{\mathrm{NF}, 2}^{\mathrm{dir}}(x) .
$$

Let us insert the full system of hadron states in the factorizable part. We then obtain

$$
\begin{aligned}
& \Pi_{\bar{b} u}(x) \equiv\left\langle T\left\{j_{\bar{b} u}(x) j_{\bar{b} u}^{\dagger}(0)\right\}\right\rangle=\sum_{h_{\bar{b} u}} R_{\bar{b} u}(x), \\
& \Pi_{\bar{c} d}(x) \equiv\left\langle T\left\{j_{\bar{c} d}(x) j_{\bar{c} d}^{\dagger}(0)\right\}\right\rangle=\sum_{h_{\bar{c} d}} R_{\bar{c} d}(x),
\end{aligned}
$$

where $R_{\bar{b} u}(x)$ and $R_{\bar{c} d}(x)$ are the quantities coming from hadron saturation, the explicit form of which is irrelevant. Important for us is the fact that the sum runs over the full system of hadron states with flavors $\bar{b} u\left(h_{\bar{b} u}\right)$ and $\bar{c} d\left(h_{\bar{c} d}\right)$, respectively. Consequently, the system of the intermediate hadron states that emerges in the factorizable part of $\Pi^{\text {dir }}$ is just the direct product of these two systems, $h_{\bar{b} u} \otimes h_{\bar{c} d}$. No other hadron state, in particular, no exotic state, may 


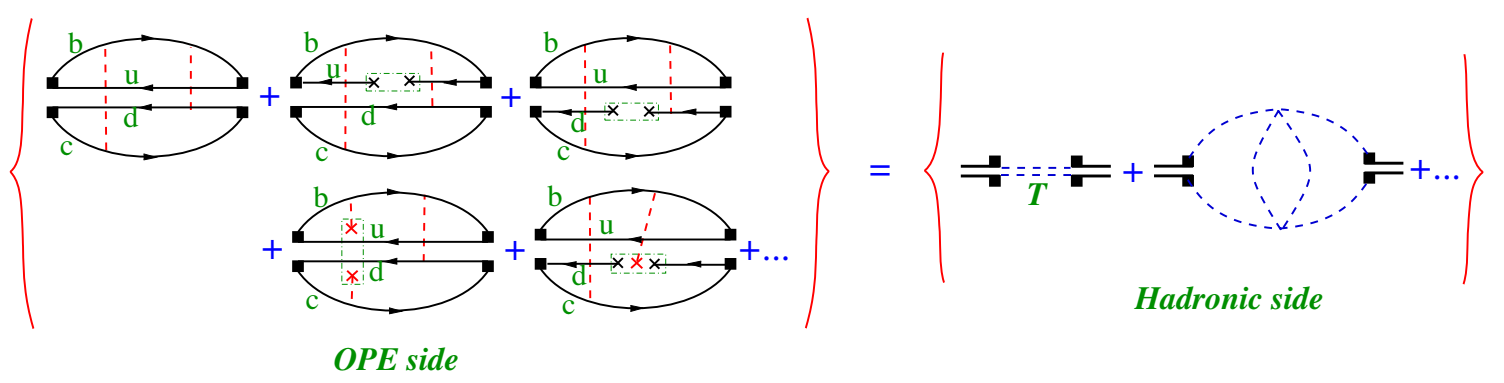

FIG. 9. T-adequate sum rule that emerges after the exact cancellations of factorizable contributions on the OPE side vs the hadronic side have been taken into account. The OPE side contains nonfactorizable diagrams with two gluon exchanges between the quark loops $b u$ and $c d$. A typical contribution with gluon exchanges between the loops, joining $b c$ and $b d$ quark pairs, and the corresponding condensate corrections are explicitly shown. [It is noteworthy that all appropriate condensate contributions are obtained according to the known rules [3] (i.e., by breaking the lines of light quarks and gluons) from the perturbative nonfactorizable Feynman diagrams with two or more gluon exchanges between the loops; no other condensate contributions appear.] The dots stand for other two-gluon exchanges (i.e., joining $u c$ and $u d, b c$ and $u d, u c$ and $b d$ pairs from the different loops). All nonfactorizable diagrams scale as $N_{c}^{0}$. The hadronic side contains the assumed tetraquark contribution and nonfactorizable meson interaction diagrams.

contribute here. So, we conclude that an exotic state, if it exists in the hadron spectrum of $\bar{b} u \bar{c} d$ states, contributes only to the nonfactorizable part of an exotic correlation function.

\section{D. $T$-adequate sum rule and the couplings of tetraquark bound states to tetraquark currents}

The $T$-adequate sum rule emerges after Eqs. (3.6) and (3.7) have been taken into account, leading to exact cancellations between the factorizable $O\left(N_{c}^{2}\right)$ contributions on the OPE side and the hadron side of the duality relation for the direct two-point function $\Pi^{\mathrm{dir}}$. Figure 9 shows the corresponding $T$-adequate sum rule: its OPE side contains nonfactorizable diagrams of order $O\left(N_{c}^{0}\right)$ (both perturbative and condensate contributions); its hadron side contains the assumed tetraquark contribution and nonfactorizable meson diagrams. The contribution of the tetraquark $T$ of flavor content $\bar{b} \bar{c} u d$ and mass $M$ to the hadronic side in momentum space has the form ${ }^{2}$

$$
f_{T}^{2} \frac{1}{M^{2}-p^{2}}
$$

and is expressed via the tetraquark coupling to the interpolating tetraquark current:

\footnotetext{
${ }^{2}$ To be more precise, there are two tetraquark currents of global $\bar{b} \bar{c} u d$ flavor content: $\theta_{\bar{b} u \bar{c} d}$ and $\theta_{\bar{b} d \bar{c} u}$ [Eq. (3.1)]. Respectively, there might be two tetraquark states of the $\bar{b} \bar{c} u d$ flavor content, $T_{1}$ and $T_{2}: T_{1}$ couples more strongly to the $\theta_{\bar{b} u \bar{c} d}$ current (the corresponding coupling scales as $N_{c}^{0}$ ) and more weakly to the $\theta_{\bar{b} d \bar{c} u}$ current (the corresponding coupling scales as $1 / N_{c}$ ), and vice versa for $T_{2}$. So, the contribution of $T_{1}$ to the correlation function (3.2) scales like $N_{c}^{0}$, whereas the contribution of $T_{2}$ is suppressed and scales like $1 / N_{c}^{2}$. These subtleties are, however, a bit outside the main discussion of this paper, so we refer, for details, to Sec. 2 of [20].
}

$$
f_{T}=\left\langle 0\left|\theta_{\bar{b} u \bar{c} d}\right| T\right\rangle
$$

Obviously, the $T$-adequate sum rule implies $f_{T} \sim N_{c}^{0}$. This feature is in full agreement with the known property of large- $N_{c}$ QCD that only noninteracting ordinary mesons saturate the $N_{c}$-leading QCD diagrams. We emphasize once more that for the consideration of exotic states, the factorizable part of the OPE is irrelevant.

Moreover, we would like to point out the following qualitative difference between the correlation functions of tetraquarks versus those of bilinear quark currents: as we have discussed, the existence of stable vector mesons in the hadron spectrum at large $N_{c}$ is required by matching the large- $N_{c}$ behavior of the OPE side and of the hadron side of the vector two-point function $\Pi^{V}$; without vector mesons populating the $O\left(N_{c}\right)$ part of the hadronic side no matching may be obtained. For the two-point functions of the tetraquark currents, the situation is qualitatively different: the factorizable OPE and hadronic sides match each other just due to the duality relations for the two-point functions of the bilinear currents. The nonfactorizable OPE side and its nonfactorizable hadronic side have the same large- $N_{c}$ behavior with or without the tetraquark bound state. So, the existence of narrow tetraquark hadrons cannot be established merely on the basis of the large- $N_{c}$ behavior of the exotic Green functions; large- $N_{c}$ QCD does not exclude narrow exotic states in $N_{c}$-subleading parts of the Green functions, but also remains consistent if such exotic states do not exist in the hadron spectrum.

\section{CONCLUSIONS AND OUTLOOK}

We discussed in great detail the OPE for two-point Green functions of the bilinear and quadrilinear colorless quark currents at large $N_{c}$ and emphasized the qualitative differences between these two objects: 
(i) In the case of two-point functions of bilinear quark currents, the contributions of single-meson states with appropriate quantum numbers emerge in the $N_{c}$-leading part of the Green function. Matching the large- $N_{c}$ behavior of the OPE series and of the hadron saturation series requires the existence of stable mesons with large couplings, $f_{V} \sim \sqrt{N_{c}}$, in the limit $N_{c} \rightarrow \infty$. The typical QCD sum rule then relates the $N_{c}$-leading $O\left(N_{c}\right)$ part of the OPE to the $O\left(N_{c}\right)$ part of the sum over hadron states, and is therefore fully consistent at large $N_{c}$.

(ii) In the case of two-point functions of tetraquark currents, the $N_{c}$-leading part of the OPE factorizes into a product of two colorless clusters. Each of them is saturated by the ordinary hadrons that may emerge in the quark-antiquark correlation functions with appropriate quark-flavor content. As a result, tetraquark states (whatever generalization of the $N_{c}=3$ tetraquark to $N_{c} \neq 3$ is considered) cannot contribute to the $N_{c}$-leading parts of the Green functions. This property fully agrees with the well-known rigorous property of large- $N_{c}$ QCD: $N_{c}$-leading Green functions are fully saturated by noninteracting ordinary mesons. The contribution of any colorless state with a more complicated quark structure, for instance, of an exotic meson, may only appear in $N_{c}$-subleading nonfactorizable parts of the Green functions of tetraquark currents. Moreover, this property is perfectly satisfied by the $T$-adequate QCD sum rules formulated in [21,22]: one of the outcomes of the $T$-adequate sum rules is the scaling of the tetraquark coupling to the tetraquark current $f_{T} \sim N_{c}^{0}$. In the present paper, we have complemented our previous analysis with a detailed discussion of the vacuum condensate contributions.
Existing typical applications of QCD sum rules to the analysis of the tetraquark candidates (see, e.g., recent publications [27-29]) relate the tetraquark properties to the low-energy part of the factorizable two-point Green functions of the tetraquark currents. Such an approach copies the route of the sum-rule analysis of the ordinary mesons and does not take into account the fundamental differences between the correlation functions of the bilinear quark currents and of the tetraquark currents. The tetraquark properties are then extracted exclusively from those parts of QCD Green functions which do not have tetraquarks as intermediate states [27-29], a feature which does not seem physically meaningful to us. Also, one can easily take the large- $N_{c}$ limit of the corresponding sum-rule analytic expressions for the couplings to immediately find that $f_{T} \sim N_{c}$. This would mean that, in contradiction to the rigorous property of $\mathrm{QCD}$ at large $N_{c}$, tetraquark poles appear in the $N_{c}$-leading QCD diagrams. $T$-adequate sum rules of $[21,22]$, now complemented with the appropriate account of condensate contributions, are free from these shortcomings and lead to fully consistent relations.

\section{ACKNOWLEDGMENTS}

D. M. and H.S. are grateful for support under joint CNRS/RFBR Grant No. PRC Russia/19-52-15022. H.S. acknowledges support from the EU research and innovation program Horizon 2020, under Grant Agreement No. 824093. D. M. would like to thank the Organizers of the MIAPP program "Deciphering Strong-Interaction Phenomenology through Precision Hadron-Spectroscopy" held in October 2019 at the Excellence Cluster "Universe" in Garching, Germany, for financial support of his participation at this workshop, where a part of this work was presented.
[1] K. G. Wilson, Non-Lagrangian models of current algebra, Phys. Rev. 179, 1499 (1969).

[2] M. A. Shifman, A. I. Vainshtein, and V. I. Zakharov, QCD and resonance physics. Theoretical foundations, Nucl. Phys. B147, 385 (1979).

[3] V. A. Novikov, M. A. Shifman, A. I. Vainshtein, and V. I. Zakharov, Calculations in external fields in quantum chromodynamics. Technical review, Fortsch. Phys. 32, 585 (1984).

[4] V. A. Novikov, M. A. Shifman, A. I. Vainshtein, and V. I. Zakharov, Wilson's operator expansion: Can it fail?, Nucl. Phys. B249, 445 (1985).

[5] B. L. Ioffe, QCD at low energies, Prog. Part. Nucl. Phys. 56, 232 (2006).
[6] G. 't Hooft, A planar diagram theory for strong interactions, Nucl. Phys. B72, 461 (1974).

[7] E. Witten, Baryons in the $1 / N$ expansion, Nucl. Phys. B160, 57 (1979).

[8] S. Coleman, Aspects of Symmetry (Cambridge University Press, Cambridge, England, 1985), Chap. 8.

[9] J. R. Pelaez, On the Nature of Light Scalar Mesons from Their Large- $N_{c}$ Behavior, Phys. Rev. Lett. 92, 102001 (2004).

[10] S. Weinberg, Tetraquark Mesons in Large- $N$ Quantum Chromodynamics, Phys. Rev. Lett. 110, 261601 (2013).

[11] F.-K. Guo, L. Liu, U.-G. Meissner, and P. Wang, Tetraquarks, hadronic molecules, meson-meson scattering and disconnected contributions in lattice QCD, Phys. Rev. D 88, 074506 (2013). 
[12] M. Knecht and S. Peris, Narrow tetraquarks at large $N$, Phys. Rev. D 88, 036016 (2013).

[13] T. D. Cohen and R. F. Lebed, Are there tetraquarks at large $N_{c}$ in QCD(F)?, Phys. Rev. D 90, 016001 (2014).

[14] T. Cohen, F. J. Llanes-Estrada, J. R. Peláez, and J. R. de Elvira, Nonordinary light meson couplings and the $1 / N_{c}$ expansion, Phys. Rev. D 90, 036003 (2014).

[15] L. Maiani, A. D. Polosa, and V. Riquer, Tetraquarks in the $1 / N$ expansion and meson-meson resonances, J. High Energy Phys. 06 (2016) 160.

[16] W. Lucha, D. Melikhov, and H. Sazdjian, Narrow exotic tetraquark mesons in large- $N_{c}$ QCD, Phys. Rev. D 96, 014022 (2017).

[17] W. Lucha, D. Melikhov, and H. Sazdjian, Tetraquark and two-meson states at large $N_{c}$, Eur. Phys. J. C 77, 866 (2017).

[18] L. Maiani, A. D. Polosa, and V. Riquer, Tetraquarks in the 1/ $N$ expansion: A new appraisal, Phys. Rev. D 98, 054023 (2018).

[19] L. Maiani, V. Riquer, and W. Wang, Tetraquarks, pentaquarks and dibaryons in the large $N$ QCD, Eur. Phys. J. C 78, 1011 (2018).

[20] W. Lucha, D. Melikhov, and H. Sazdjian, Are there narrow flavor-exotic tetraquarks in large- $N_{c}$ QCD?, Phys. Rev. D 98, 094011 (2018).
[21] W. Lucha, D. Melikhov, and H. Sazdjian, Tetraquarkadequate formulation of QCD sum rules, Phys. Rev. D 100, 014010 (2019).

[22] W. Lucha, D. Melikhov, and H. Sazdjian, Tetraquarkadequate QCD sum rules for quark-exchange processes, Phys. Rev. D 100, 074029 (2019).

[23] H.E. Haber, Useful relations among the generators in the defining and adjoint representations of $\mathrm{SU}(N)$, arXiv: 1912.13302.

[24] W. Lucha, D. Melikhov, and H. Sazdjian, Cluster reducibility of multiquark operators, Phys. Rev. D 100, 094017 (2019).

[25] R. L. Jaffe, Multi-quark hadrons. 2. Methods, Phys. Rev. D 15, 281 (1977).

[26] R. L. Jaffe, Two types of hadrons, Nucl. Phys. A804, 25 (2008).

[27] A. Pimikov, H.-J. Lee, and P. Zhang, Hidden charm pentaquarks with color-octet substructure in QCD sum rules, Phys. Rev. D 101, 014002 (2020).

[28] Z.-G. Wang, Landau equation and QCD sum rules for the tetraquark molecular states, Phys. Rev. D 101, 074011 (2020).

[29] R. M. Albuquerque, S. Narison, A. Rabemananjara, D. Rabetiarivony, and G. Randriamanatrika, Doubly-hidden scalar heavy molecules and tetraquarks states from QCD at NLO, Phys. Rev. D 102, 094001 (2020). 